\title{
Peripheral alpha-synuclein levels in patients with genetic and non- genetic forms of Parkinson's Disease
}

\author{
Evangelia Emmanouilidou $^{1,2}$, Nikolaos Papagiannakis ${ }^{3,4,5}$, Stella Kouloulia ${ }^{6}$, \\ Aikaterini Galaziou ${ }^{6}$, Roubina Antonellou ${ }^{5}$, Dimitra Papadimitriou ${ }^{7}$, Aglaia \\ Athanasiadou ${ }^{8}$, Maria Bozi ${ }^{5}$, Christos Koros ${ }^{4}$, Matina Maniati ${ }^{3}$, Kostas \\ Vekrellis ${ }^{1}$, Penelope C. Ioannou ${ }^{6}$, Leonidas Stefanis ${ }^{3,4,5 *}$
}

${ }^{1}$ Center of Basic Research, Biomedical Research Foundation of the Academy of Athens, Athens, Greece

2 Laboratory of Biochemistry, Department of Chemistry, National and Kapodistrian University of Athens, Athens, Greece

${ }^{3}$ Center of Clinical Research, Experimental Surgery and Translational Research, Biomedical Research Foundation of the Academy of Athens, Athens, Greece

${ }^{4}$ First Department of Neurology, National and Kapodistrian University of Athens Medical School, Athens, Greece

${ }^{5}$ Second Department of Neurology, National and Kapodistrian University of Athens Medical School, Athens, Greece

${ }^{6}$ Laboratory of Analytical Chemistry, Department of Chemistry, National and Kapodistrian University of Athens

${ }^{7}$ Department of Neurology, Henry Dunant Hospital Center

${ }^{8}$ Department of Biology, University of Patras Medical School

*Corresponding author

Center of Clinical Research, Experimental Surgery and Translational Research

Biomedical Research Foundation of the Academy of Athens (BRFAA)

4 Soranou Efesiou Str, Athens 11527, Greece

Tel: +30 2106597214, Fax: +30 2106597545, e-mail: 1stefanis@bioacademy.gr

Word count: 2998

Keywords: Parkinson Disease; alpha-synuclein; Plasma; Serum; A53T.

Funding: This study was supported in part by grants to LS from the Hellenic Ministry of Education and Religion THALIS grant number 377206 and from the EU Program MEFOPA (FP7-HEALTH-241791). 


\section{$\underline{\text { Abstract }}$}

Background: Variations of $\alpha$-synuclein levels have been reported in serum and plasma in Parkinson's Disease (PD) Patients.

Methods: Serum and plasma were obtained from PD patients without known mutations (GU-PD, $n=124)$ ), carriers of the A53T/G209A point mutation in the $\alpha$ synuclein gene $(S N C A)(\mathrm{n}=29)$, and respective age-/sex-matched controls. Levels of total $\alpha$-synuclein were assessed using an in-house ELISA assay.

Results: A statistically significant increase of $\alpha$-synuclein levels was found in serum, but not plasma, from GU-PD patients compared to healthy controls. A statistically significant decrease of $\alpha$-synuclein levels was found in serum and plasma from symptomatic A53T mutation carriers compared to healthy controls. Plasma $\alpha$ synuclein levels were modestly negatively correlated with UPDRS part III score and disease duration in A53T-PD patients.

Conclusion: Increased $\alpha$-synuclein levels in serum of GU-PD patients suggest a systemic deregulation of $\alpha$-synuclein homeostasis in PD. The opposite results in A53T-PD highlight the complexity of $\alpha$-synuclein homeostatic regulation in PD, and suggest the possibility of reduced expression of the mutant allele. 


\section{Introduction}

Parkinson's disease (PD) represents the second most common neurodegenerative disease, characterized by the relatively selective degeneration of the dopaminergic neurons in the Substantia nigra pars compacta, although other brain regions also participate in the degenerative process. The surviving neurons contain intracellular proteinacious inclusions, Lewy bodies (LBs) and Lewy neurites (LNs), rich in filamentous protein structures positive for $\alpha$-synuclein [1]. The diagnosis of PD is clinical, and no reliable biomarker, with the possible exception of expensive dopamine transporter imaging, has emerged for the diagnosis of PD (especially in its premotor phase), for disease progression, or for differential diagnosis [2]. There is a need therefore for the development of such biomarkers, through a better understanding of disease pathogenesis.

Alpha-synuclein is a highly conserved neuronal presynaptic protein of 140 amino acids, whose levels, along with its accumulation in $\beta$-sheet rich oligomers and more mature aggregates in the form of LBs and LNs, are thought to be critical to PD pathogenesis [3]. This notion is further supported by the fact that point mutations as well as duplications and triplications of the $S N C A$ locus encoding for $\alpha$-synuclein lead to the rare cases of familial PD, while Genome-Wide Association Studies (GWAS) have implicated the SNCA locus in sporadic PD [4]. Apart from its predominant localization within neuronal cells in the peripheral (PNS) and central nervous system (CNS), $\alpha$-synuclein can be detected as an extracellular protein in the cerebrospinal fluid (CSF), as well as the brain interstitial fluid, following secretion from neuronal cells [5]. Furthermore, $\alpha$-synuclein can be detected in peripheral fluids and cells, such as plasma, serum, erythrocytes and platelets [6-8]. The direct genetic and biochemical link of $\alpha$-synuclein to PD pathogenesis and the ability to quantify its levels in a plethora of biological fluids render $\alpha$-synuclein a promising potential biomarker for PD and related disorders.

Most studies have focused on the measurement of $\alpha$-synuclein levels in CSF, and a consistent finding has been that such levels are decreased in the context of PD, likely due to the deposition of $\alpha$-synuclein in aggregates, limiting the soluble, secreted species of the protein [9]. However, differences from controls are relatively small, and assays for total $\alpha$-synuclein in CSF are on their own not able to reliably differentiate 
PD patients from controls [9]. More recent CSF studies using amplification methods such as RT-QuIC and PMCA report a high sensitivity and specificity for the differentiation of PD patients from controls [10]. However, such studies require specialized equipment and reagents, and involve the invasive procedure of lumbar puncture.

Analysis of peripheral $\alpha$-synuclein includes the detection of the monomeric and oligomeric forms of $\alpha$-synuclein in blood plasma [7], serum [7], PBMCs [11], erythrocytes [12] or saliva [13]. The studies conducted so far have not produced conclusive results. Available studies are contradictory, reporting an increase [14,15] or a decrease $[16,17]$ in plasma total $\alpha$-synuclein levels in PD patients compared to controls. Likewise, plasma oligomeric $\alpha$-synuclein levels have been reported to be increased in PD [14], or to be no different from controls [7].

The majority of studies provide measurement of peripheral $\alpha$-synuclein in different cohorts of sporadic PD patients. In the current study, we have determined the levels of monomeric total $\alpha$-synuclein in the plasma and serum of a defined cohort of idiopathic PD patients and control subjects but also in a cohort of PD patients harboring the p.G209A/A53T mutation. The p.G209A/A53T (rs104893877) point mutation in SNCA was the first mutation described in familial cases of PD [18]. The use of this rare cohort of A53T $\alpha$-synuclein carriers, a mutation of high penetrance, allowed us to compare the results obtained from idiopathic PD patients with patients possessing a defined genetic background that leads to the development of PD pathology.

Even though our group has previously reported that $\alpha$-synuclein levels in erythrocyte membranes remain unaffected in A53T mutation carriers compared to healthy subjects [19], the direct measurement of plasma/serum $\alpha$-synuclein levels in these patients has not been addressed. The assessment of both blood plasma and serum was selected in order to account for possible interactions of the targeted protein with coagulation factors and reveal potential correlations in the two biological fluids that are mostly used for the measurement of peripheral $\alpha$-synuclein.

\section{Materials and Methods}

\section{Subjects}


Patients with PD and age-matched healthy subjects were enrolled from Attikon University Hospital, Athens, Greece (Table 1). Subjects harboring the G209A/A53T mutation in the SNCA gene and their respective age-matched healthy controls (Table 2), were separately recruited in the MEFOPA study (MEndelian FOrms Of Parkinsonism) [20]. The subjects were divided accordingly into two categories: on the one hand, patients without the A53T mutation in the SNCA gene, nor mutations in the GBA gene (Genetically-Undetermined PD patients, GU-PD) [20], and, on the other, A53T mutation carriers (A53T-PD). The SNCA A53T mutation and GBA mutations are the most common causes of genetic PD in Greece, other causes of monogenic PD, including LRRK2 mutations, being extremely rare in this population [20]. Therefore, for the GU-PD group, we only assessed and excluded carriers of these two types of mutations. Of note, all carriers of the A53T SNCA mutation tested negative for GBA mutations. Enrolled healthy controls had no known family history of PD. They were not screened for any of the aforementioned mutations.

All study procedures were approved by the scientific council and ethical committee of Attikon Hospital and all participants provided written informed consent.

\section{Clinical Assessment}

Patients' motor condition was evaluated with the use of the Unified Parkinson's Disease Rating Scale (UPDRS) Part III (in the on phase in the case of motor fluctuations) and their cognitive function was measured with the Mini-Mental State Examination (MMSE) or, in the case of the p.A53T cohort, with the MOCA (Tables 1 and 2). Clinical assessments were conducted the same day that blood samples were collected.

\section{Collection of serum and plasma}

Blood was collected in EDTA- and clot activator-coated tubes (BD Vacutainer, Ref \#366643 and \#367986 respectively. Each sample was centrifuged at $2200 \mathrm{~g}$ for 10 minutes and the supernatant was collected, containing plasma or serum depending on the vial used. Plasma and serum were aliquoted in $2 \mathrm{ml}$ polypropylene tubes and stored at $-80^{\circ} \mathrm{C}$.

\section{Detection of the p.A53T/c.G209A mutation}


DNA was extracted from peripheral blood and was analyzed for the presence of the SNCA-G209A mutation as described previously by Bozi and colleagues [20].

\section{Quantification of $\alpha$-synuclein levels in serum and plasma}

The levels of $\alpha$-synuclein in serum and plasma were quantified using our in house ELISA as previously described [5,21]. Samples were diluted 60x in TBST buffer $(10$ $\mathrm{mM}$ Tris-Cl, $\mathrm{pH}$ 7.6, $100 \mathrm{mM} \mathrm{NaCl}, 0.1 \%$ Tween-20) and assessed in quadruplicate using the standard addition method. Each sample was measured alone and spiked with two different additions of recombinant $\alpha$-synuclein at final concentrations of $0.1,0.3$ $\mathrm{ng} / \mathrm{ml}$ and $0.9,2.7 \mathrm{ng} / \mathrm{ml}$, in plasma and serum, respectively. The final concentration for each sample was estimated as the mean value of the concentration estimated following spiking of the two concentrations of the standard. The group of samples from A53T patients and their controls were measured in the Biomedical Research Foundation of the Academy of Athens during 2012 - 2013. The group of GU-PD and the respective control group were measured during 2013 - 2015 in the Laboratory of Analytical Chemistry, University of Athens using a different chemiluminescence reader, which explains the differences we observe in the absolute concentration values between the two cohorts. It is for this reason that we established a separate control group for each cohort of PD patients.

\section{Statistical analysis}

Statistical analysis was performed using R version 3.5.3 with RStudio. We have used the $\mathrm{W}$ test statistic, to assess differences between groups. All data underwent a normality test (Shapiro-Wilk), and were found to be non-normally distributed. Results were reported mentioning the $\mathrm{W}$ statistic of the non-parametric MannWhitney test and the respective p-value as computed by R. Spearman's method was used to correlate $\alpha$-synuclein levels with participants' demographic and clinical characteristics. The threshold for significance was set to p-values less than 0.05 . The cutoff for the ROC analysis was determined using the Youden Index.

\section{$\underline{\text { Results }}$}


We initially analyzed the idiopathic PD group $(\mathrm{N}=124)$ in comparison with the control group $(\mathrm{N}=68)$ consisting of individuals not suffering from any neurological condition. The demographic and clinical profiles of these groups are described in Table 1. Measurement of $\alpha$-synuclein levels in the serum samples revealed a statistically significant increase in $\alpha$-synuclein levels in PD patients vs. healthy controls $(\mathrm{W}=2709, \mathrm{P}=0.003$ ) (Fig. 1A). Similar assessment of plasma $\alpha$-synuclein levels showed no significant differences (Fig. 1B). We found a positive correlation between $\alpha$-synuclein levels in plasma and serum in the idiopathic PD and control groups (rho=0.272, p=0.003) (Fig. 1C). Disease duration, UPDRS part III, MMSE scores or Levodopa Equivalent doses were not correlated with $\alpha$-synuclein plasma or serum levels. Given the difference in sex distribution between the control and PD groups (Table 1), we also performed analyses with sex as a confounder, which did not alter our results (data not shown).

ROC analysis was performed between healthy controls and PD patients, which were age- and sex-matched, for assessing the diagnostic utility of serum $\alpha$-synuclein levels. The area under the curve is $0.632(95 \%$ confidence intervals $0.546-0.718)$. A cutoff value for the serum $\alpha$-synuclein of $45.6 \mathrm{ng} / \mathrm{ml}$ was selected, yielding a sensitivity of $58.3 \%$ (CI: $48.7 \%-67.3 \%$ ) and a specificity of $62.5 \%$ (CI: $49.5 \%-74.3 \%$ ). The positive predictive value for the presence of PD was 73.6\% (CI: $62.1 \%-80.5 \%$ ) (Fig. 1D).

Next, we analyzed the group of A53T $\alpha$-synuclein mutation carriers $(N=29$, of which 6 asymptomatic and 23 symptomatic) compared to a separate group of age-matched healthy controls $(\mathrm{N}=29)$. We found a statistically significant decrease in both serum and plasma $\alpha$-synuclein levels in A53T carriers compared to the controls (serum: $\mathrm{W}=216, \mathrm{p}=0.002$; plasma: $\mathrm{W}=211, \mathrm{p}=0.008$ ) (Fig. 2A, B). Even considering symptomatic and asymptomatic carriers as separate categories, the statistically significant decrease against healthy controls is still manifested in serum for both categories (asymptomatic $\mathrm{p}=0.046$; symptomatic $\mathrm{p}=0.005$ ) and in plasma in A53T-PD patients $(\mathrm{p}<0.001)$.

Interestingly, within the A53T group, asymptomatic carriers exhibited significantly higher levels of plasma $\alpha$-synuclein (Fig. 2C). Further analysis revealed a moderate negative correlation in the A53T group between UPDRS part III and $\alpha$-synuclein levels in plasma, that however barely missed statistical significance ( $\mathrm{rho}=-0.382$, $\mathrm{p}=0.054$ ) (Fig. 2D). A moderate negative correlation between plasma $\alpha$-synuclein 
levels and the duration of $\mathrm{PD}(\mathrm{rho}=-0.396, \mathrm{p}=0.045)$ in the symptomatic A53Tcarriers subgroup was also established (Fig. 2E). No other significant correlations were identified.

In our experiments, we have consistently observed that, within control groups, serum levels of $\alpha$-synuclein were about two times higher than the commensurate plasma levels. We found that this difference was statistically significant in the controls of the GU-PD cohort $(59.96 \pm 7.43$ vs $28.30 \pm 4.23 \mathrm{ng} / \mathrm{ml}$ in the serum and plasma, respectively, $\mathrm{p}<0.0001$, Mann-Whitney non parametric t-test) and in the controls of the A53T cohort $(25.43 \pm 2.58$ vs $16.02 \pm 4.21 \mathrm{ng} / \mathrm{ml}$ in the serum and plasma, respectively, $\mathrm{p}=0.0004$, Mann-Whitney non parametric t-test). We have investigated whether the differences in the levels of plasma and serum $\alpha$-synuclein within a group could be attributed to the presence of EDTA as an anticoagulant. The presence of EDTA did not affect the performance of the ELISA assay as observed by the measurement of recombinant $\alpha$-synuclein (Fig. 3A). To further verify that EDTA does not interfere with our measurement, a serum sample was aliquoted and analyzed in the absence and in the presence of EDTA. Three aliquots of each condition (+/- EDTA) were measured using the ELISA. No differences were observed in the levels of $\alpha$ synuclein with or without EDTA (Fig. 3B).

\section{$\underline{\text { Discussion }}$}

A major result from our study is that serum, but not plasma, levels of total $\alpha$-synuclein are higher in patients with idiopathic PD compared to controls. Previous work has given contradictory results in this regard. The studies that have assessed levels of $\alpha$ synuclein in serum in PD patients have found either that they are unaffected in PD patients [22] or are decreased compared to controls in a larger study [23]. In comparison, total plasma $\alpha$-syn levels in PD patients increased in two studies using ELISA [14,15], but decreased in another using western blot [16]. Two recent studies, that measured plasma $\alpha$-syn via ELISA [24-26] and mass spectrometry [26] found similar levels in PD patients compared to controls. In larger cohorts, when total plasma $\alpha$-syn levels were assessed using Luminex assays, no difference was found between PD patients and controls [27,28]. Conversely, a recent study suggests that plasma total $\alpha$-syn levels assessed using ELISA are decreased in patients with 
sporadic PD compared to controls, with a similar trend seen with familial PD [29]. No studies, to our knowledge, have assessed $\alpha$-synuclein levels concomitantly in plasma and serum, as in the present work.

In our study, we have found significantly higher $\alpha$-synuclein levels in the serum samples of GU-PD patients vs the control subjects. Interestingly, this difference could not be reproduced in the respective plasma samples of this cohort which also exhibited significantly lower levels of $\alpha$-synuclein compared with the serum samples by approximately two-fold. Serum and plasma have been widely used as biological matrices to assess the presence of proteins or metabolites that could assist clinical diagnosis. However, it has been reported that the selection of the blood fraction could have important implications regarding the concentration of the analyte measured and, consequently, the interpretation of the produced results [30,31]. To obtain serum, the products of the coagulation cascade along with blood cells are removed from the sample by centrifugation. During this procedure, proinflammatory cytokines and specific metabolites are released by platelets into the sample [32,33]. To obtain plasma, coagulation is prevented by the addition of EDTA (as used here) or heparin. In some cases, the variability in the measurements between serum and plasma is due to the EDTA-induced chelation of metal ions that ensure sufficient epitope detection in ELISA assays. In the current study, we have ruled out the possibility that the EDTA present in the plasma collection material could act as inhibitor in the performed ELISA assay. However, we cannot rule out the possibility that the lower $\alpha$ synuclein concentration in our plasma samples results from modifications in the folding properties of $\alpha$-synuclein due to its inefficient binding to metal ions. This was also true for other proteins measured in serum and plasma samples such as the RACEbinding protein, BDNF and metalloproteinases 1, 7 and 10 [30]. In sum, our data indicate that the choice of the blood fraction is critical for the assessment of $\alpha$ synuclein concentration.

These results are consistent with the notion, reinforced by GWAS and other studies linking SNCA haplotypes with peripheral levels of $\alpha$-synuclein [34,28], that, in PD patients, various factors converge onto leading to slightly higher systemic levels of $\alpha$ synuclein and thus increase the risk of developing PD. It should be stressed that such differences in $\alpha$-synuclein levels are of small magnitude, and are not useful as biomarker indices; indeed, the sensitivity and specificity of high serum $\alpha$-synuclein levels we have obtained in the current study are relatively low, and unlikely to be used 
in clinical practice, at least without additional factors. The lack of a difference in $\alpha$ synuclein levels in peripheral body fluids in many other studies may be due to type II error, given the fact that in many cases smaller numbers of subjects were ascertained, and only plasma was assessed. It should be stressed here that total $\alpha$-synuclein may not accurately reflect the concentration of pathogenic forms of this protein. Studies assessing aberrant conformations of $\alpha$-synuclein, such as oligomeric or phosphorylated forms, may be more revealing in this regard.

Few biomarker studies have been performed in genetic synucleinopathy cohorts, given the rarity of the condition. We have previously reported that levels of $\alpha$ synuclein in erythrocyte membranes, as assessed by western immunoblotting, were not different in p.A53T PD patients compared to controls [19]. However, in the current study, using a more sensitive ELISA technique, we have found that actually, in both serum and plasma, levels of $\alpha$-synuclein are lower in this group of patients compared to controls. This appeared to depend on disease status, as asymptomatic carriers had higher levels that approximated those of controls, while there was also a significant, albeit moderate, negative correlation between plasma values and disease duration and a trend in the same direction between plasma values and UPDRS III that barely failed to reach statistical significance. These data suggest that the existence, duration and severity of the disease may be a factor that leads to the lowering of $\alpha$ synuclein levels in this group of subjects. In fact, the present study raises the interesting possibility that in future longitudinal studies in asymptomatic A53T mutation carriers declining peripheral levels of $\alpha$-synuclein may serve as a marker of transition from the asymptomatic to the symptomatic phase.

Indeed, it has been previously reported, based on lymphoblastoid cell lines, that mRNA and protein levels of $\alpha$-synuclein derived from the mutant allele are reduced in carriers of the p.A53T and p.A30P mutations, in a manner dependent on the duration and severity of the disease $[35,36]$. The mechanism for this effect is unclear, but may involve epigenetic histone modifications that lead to reduction in the expression of the mutant allele [37]. It is likely that such reduced expression of the mutant allele may also occur systemically in such patients, leading to lower levels of total $\alpha$-synuclein in the present study. In fact, lower levels of $\alpha$-synuclein derived from the mutant allele might explain the lack of an increase in dimeric species of $\alpha$-synuclein in erythrocyte membranes of such patients [19], which was unexpected given the increased propensity of the mutant p.A53T form to oligomerize and aggregate [38]. It will be 
very interesting to assess mRNA and protein levels of $\alpha$-synuclein, and in particular of the mutant allele, in the brains of patients with the p.A53T mutation, given the demonstrated aggregation of the protein in such post-mortem material [39]. Alternative explanations for the lower $\alpha$-synuclein levels in this group of patients include decreased secretion or increased turnover of the mutant allele. However, there is no indication that the A53T mutant is secreted less than the WT, at least in cellular systems (unpublished data from our laboratories), and, if anything, the halflife of the A53T mutant is longer than that of the WT form [40,41].

Limitations of our study include the relatively small sample size, especially for the A53T arm of the study, and the fact that a complete PD-related mutation status was not assessed in all subjects, including controls. However, given the rarity of PDrelated mutations in the general population, especially regarding the $\mathrm{p}$. A53T SNCA mutation, we do not believe that this issue could have influenced our results. It would have been optimal to also assess peripheral $\alpha$-synuclein levels in PD patients with GBA mutations, however we could not collect sufficient samples from such patients for the present study. It would be important in future studies to assess peripheral $\alpha$ synuclein markers in this group of subjects, as it is largely considered to represent another type of synucleinopathy; on the other hand, other SNCA point mutation, as well as multiplication, carriers, need to also be systematically assessed in this regard. In conclusion, we show here that serum, but not plasma, levels of $\alpha$-synuclein are increased in the blood of patients with PD compared to controls, in results that are broadly in agreement with the $\alpha$-synuclein burden hypothesis. It should be stressed that such differences are small and unlikely to be useful in clinical practice. The opposite effect, a decrease in total $\alpha$-synuclein levels in plasma and serum, is observed in subjects with the p.A53T mutation in the SNCA gene, in a manner partially dependent on the presence, duration and severity of the disease, suggesting that this effect may be associated with the previously reported decreased expression of the mutant allele. These disparate effects underline the complexity of the involvement of $\alpha$-synuclein in PD, and raise the possibility that different pathogenetic mechanisms may drive the neurodegenerative process in genetic and sporadic PD.

\section{$\underline{\text { References }}$}


[1] M.G. Spillantini, R.A. Crowther, R. Jakes, M. Hasegawa, M. Goedert, alphaSynuclein in filamentous inclusions of Lewy bodies from Parkinson's disease and dementia with lewy bodies, Proceedings of the National Academy of Sciences of the United States of America. 95 (1998) 6469-73.

[2] S. Lotankar, K.S. Prabhavalkar, L.K. Bhatt, Biomarkers for Parkinson's Disease: Recent Advancement, Neurosci Bull. 33 (2017) 585-597. https://doi.org/10.1007/s12264-017-0183-5.

[3] M.R. Cookson, M. van der Brug, Cell systems and the toxic mechanism(s) of alpha-synuclein, Experimental Neurology. 209 (2008) 5-11. https://doi.org/10.1016/j.expneurol.2007.05.022.

[4] J.T. Bendor, T.P. Logan, R.H. Edwards, The function of alpha-synuclein, Neuron. 79 (2013) 1044-66. https://doi.org/10.1016/j.neuron.2013.09.004.

[5] E. Emmanouilidou, D. Elenis, T. Papasilekas, G. Stranjalis, K. Gerozissis, P.C. Ioannou, K. Vekrellis, Assessment of $\alpha$-synuclein secretion in mouse and human brain parenchyma, PLoS ONE. 6 (2011) e22225. https://doi.org/10.1371/journal.pone.0022225.

[6] R. Barbour, K. Kling, J.P. Anderson, K. Banducci, T. Cole, L. Diep, M. Fox, J.M. Goldstein, F. Soriano, P. Seubert, T.J. Chilcote, Red blood cells are the major source of alpha-synuclein in blood, Neuro-Degenerative Diseases. 5 (2008) 55-9. https://doi.org/10.1159/000112832.

[7] O.M. El-Agnaf, S.A. Salem, K.E. Paleologou, M.D. Curran, M.J. Gibson, J.A. Court, M.G. Schlossmacher, D. Allsop, Detection of oligomeric forms of alphasynuclein protein in human plasma as a potential biomarker for Parkinson's disease, FASEB Journal : Official Publication of the Federation of American Societies for Experimental Biology. 20 (2006) 419-25. https://doi.org/10.1096/fj.03-1449com.

[8] Q.X. Li, B.C. Campbell, C.A. McLean, D. Thyagarajan, W.P. Gai, R.M. Kapsa, K. Beyreuther, C.L. Masters, J.G. Culvenor, Platelet alpha- and gammasynucleins in Parkinson's disease and normal control subjects, Journal of Alzheimer's Disease : JAD. 4 (2002) 309-15.

[9] L. Gao, H. Tang, K. Nie, L. Wang, J. Zhao, R. Gan, J. Huang, R. Zhu, S. Feng, Z. Duan, Y. Zhang, L. Wang, Cerebrospinal fluid alpha-synuclein as a biomarker for Parkinson's disease diagnosis: a systematic review and metaanalysis, International Journal of Neuroscience. 125 (2015) 645-654. https://doi.org/10.3109/00207454.2014.961454.

[10] G. Fairfoul, L.I. McGuire, S. Pal, J.W. Ironside, J. Neumann, S. Christie, C. Joachim, M. Esiri, S.G. Evetts, M. Rolinski, F. Baig, C. Ruffmann, R. WadeMartins, M.T.M. Hu, L. Parkkinen, A.J.E. Green, Alpha-synuclein RT-QuIC in the CSF of patients with alpha-synucleinopathies, Ann Clin Transl Neurol. 3 (2016) 812-818. https://doi.org/10.1002/acn3.338.

[11] S. Kim, B.S. Jeon, C. Heo, P.S. Im, T.-B. Ahn, J.-H. Seo, H.-S. Kim, C.H. Park, S.H. Choi, S.-H. Cho, W.J. Lee, Y.-H. Suh, Alpha-synuclein induces apoptosis by altered expression in human peripheral lymphocyte in Parkinson's disease, FASEB J. 18 (2004) 1615-1617. https://doi.org/10.1096/fj.04-1917fje.

[12] C. Tian, G. Liu, L. Gao, D. Soltys, C. Pan, T. Stewart, M. Shi, Z. Xie, N. Liu, T. Feng, J. Zhang, Erythrocytic $\alpha$-Synuclein as a potential biomarker for Parkinson's disease, Transl Neurodegener. 8 (2019) 15. https://doi.org/10.1186/s40035-019-0155-y.

[13] J.G. Goldman, H. Andrews, A. Amara, A. Naito, R.N. Alcalay, L.M. Shaw, P. Taylor, T. Xie, P. Tuite, C. Henchcliffe, P. Hogarth, S. Frank, M.-H. Saint- 
Hilaire, M. Frasier, V. Arnedo, A.N. Reimer, M. Sutherland, C. SwansonFischer, K. Gwinn, Fox Investigation of New Biomarker Discovery, U.J. Kang, Cerebrospinal fluid, plasma, and saliva in the BioFIND study: Relationships among biomarkers and Parkinson's disease Features, Mov. Disord. 33 (2018) 282-288. https://doi.org/10.1002/mds.27232.

[14] R. Duran, F.J. Barrero, B. Morales, J.D. Luna, M. Ramirez, F. Vives, Plasma alpha-synuclein in patients with Parkinson's disease with and without treatment, Movement Disorders : Official Journal of the Movement Disorder Society. 25 (2010) 489-93. https://doi.org/10.1002/mds.22928.

[15] P.H. Lee, G. Lee, H.J. Park, O.Y. Bang, I.S. Joo, K. Huh, The plasma alphasynuclein levels in patients with Parkinson's disease and multiple system atrophy, Journal of Neural Transmission. 113 (2006) 1435-9. https://doi.org/10.1007/s00702-005-0427-9.

[16] Q.X. Li, S.S. Mok, K.M. Laughton, C.A. McLean, R. Cappai, C.L. Masters, J.G. Culvenor, M.K. Horne, Plasma alpha-synuclein is decreased in subjects with Parkinson's disease, Experimental Neurology. 204 (2007) 583-8. https://doi.org/10.1016/j.expneurol.2006.12.006.

[17] R. Ishii, T. Tokuda, H. Tatebe, T. Ohmichi, T. Kasai, M. Nakagawa, T. Mizuno, O.M. El-Agnaf, Decrease in plasma levels of alpha-synuclein is evident in patients with Parkinson's disease after elimination of heterophilic antibody interference, PloS One. 10 (2015) e0123162. https://doi.org/10.1371/journal.pone.0123162.

[18] M.H. Polymeropoulos, C. Lavedan, E. Leroy, S.E. Ide, A. Dehejia, A. Dutra, B. Pike, H. Root, J. Rubenstein, R. Boyer, E.S. Stenroos, S. Chandrasekharappa, A. Athanassiadou, T. Papapetropoulos, W.G. Johnson, A.M. Lazzarini, R.C. Duvoisin, G. Di Iorio, L.I. Golbe, R.L. Nussbaum, Mutation in the alphasynuclein gene identified in families with Parkinson's disease, Science. 276 (1997) 2045-7.

[19] N. Papagiannakis, C. Koros, M. Stamelou, A.-M. Simitsi, M. Maniati, R. Antonelou, D. Papadimitriou, G. Dermentzaki, M. Moraitou, H. Michelakakis, L. Stefanis, Alpha-synuclein dimerization in erythrocytes of patients with genetic and non-genetic forms of Parkinson's Disease, Neuroscience Letters. 672 (2018) 145-149. https://doi.org/10.1016/j.neulet.2017.11.012.

[20] M. Bozi, D. Papadimitriou, R. Antonellou, M. Moraitou, M. Maniati, D.K. Vassilatis, S.G. Papageorgiou, A. Leonardos, G. Tagaris, G. Malamis, D. Theofilopoulos, S. Kamakari, E. Stamboulis, G.M. Hadjigeorgiou, A. Athanassiadou, H. Michelakakis, A. Papadimitriou, T. Gasser, L. Stefanis, Genetic assessment of familial and early-onset Parkinson's disease in a Greek population, European Journal of Neurology: The Official Journal of the European Federation of Neurological Societies. 21 (2014) 963-8. https://doi.org/10.1111/ene.12315.

[21] E. Kapaki, G.P. Paraskevas, E. Emmanouilidou, K. Vekrellis, The diagnostic value of CSF $\alpha$-synuclein in the differential diagnosis of dementia with Lewy bodies vs. normal subjects and patients with Alzheimer's disease, PLoS ONE. 8 (2013) e81654. https://doi.org/10.1371/journal.pone.0081654.

[22] L.M. Smith, M.C. Schiess, M.P. Coffey, A.C. Klaver, D.A. Loeffler, $\alpha-$ Synuclein and anti- $\alpha$-synuclein antibodies in Parkinson's disease, atypical Parkinson syndromes, REM sleep behavior disorder, and healthy controls, PLoS ONE. 7 (2012) e52285. https://doi.org/10.1371/journal.pone.0052285. 
[23] D. Besong-Agbo, E. Wolf, F. Jessen, M. Oechsner, E. Hametner, W. Poewe, M. Reindl, W.H. Oertel, C. Noelker, M. Bacher, R. Dodel, Naturally occurring $\alpha-$ synuclein autoantibody levels are lower in patients with Parkinson disease, Neurology. 80 (2013) 169-175. https://doi.org/10.1212/WNL.0b013e31827b90d1.

[24] P.G. Foulds, P. Diggle, J.D. Mitchell, A. Parker, M. Hasegawa, M. MasudaSuzukake, D.M.A. Mann, D. Allsop, A longitudinal study on $\alpha$-synuclein in blood plasma as a biomarker for Parkinson's disease, Sci Rep. 3 (2013) 2540. https://doi.org/10.1038/srep02540.

[25] M.J. Park, S.-M. Cheon, H.-R. Bae, S.-H. Kim, J.W. Kim, Elevated levels of $\alpha-$ synuclein oligomer in the cerebrospinal fluid of drug-naïve patients with Parkinson's disease, J Clin Neurol. 7 (2011) 215-222. https://doi.org/10.3988/jcn.2011.7.4.215.

[26] P.G. Foulds, J.D. Mitchell, A. Parker, R. Turner, G. Green, P. Diggle, M. Hasegawa, M. Taylor, D. Mann, D. Allsop, Phosphorylated $\alpha$-synuclein can be detected in blood plasma and is potentially a useful biomarker for Parkinson's disease, FASEB J. 25 (2011) 4127-4137. https://doi.org/10.1096/fj.10-179192.

[27] M. Shi, C.P. Zabetian, A.M. Hancock, C. Ginghina, Z. Hong, D. Yearout, K.A. Chung, J.F. Quinn, E.R. Peskind, D. Galasko, J. Jankovic, J.B. Leverenz, J. Zhang, Significance and confounders of peripheral DJ-1 and alpha-synuclein in Parkinson's disease, Neurosci. Lett. 480 (2010) 78-82. https://doi.org/10.1016/j.neulet.2010.06.009.

[28] I.F. Mata, M. Shi, P. Agarwal, K.A. Chung, K.L. Edwards, S.A. Factor, D.R. Galasko, C. Ginghina, A. Griffith, D.S. Higgins, D.M. Kay, H. Kim, J.B. Leverenz, J.F. Quinn, J.W. Roberts, A. Samii, K.W. Snapinn, D.W. Tsuang, D. Yearout, J. Zhang, H. Payami, C.P. Zabetian, SNCA variant associated with Parkinson disease and plasma alpha-synuclein level, Arch. Neurol. 67 (2010) 1350-1356. https://doi.org/10.1001/archneurol.2010.279.

[29] A. Gorostidi, A. Bergareche, J. Ruiz-Martinez, J.F. Marti-Masso, M. Cruz, S. Varghese, M.M. Qureshi, F. Alzahmi, A. Al-Hayani, A. Lopez de Munain, O.M. El-Agnaf, Alpha-synuclein levels in blood plasma from LRRK2 mutation carriers, PloS One. 7 (2012) e52312. https://doi.org/10.1371/journal.pone.0052312.

[30] M. Alsaif, P.C. Guest, E. Schwarz, A. Reif, S. Kittel-Schneider, M. Spain, H. Rahmoune, S. Bahn, Analysis of serum and plasma identifies differences in molecular coverage, measurement variability, and candidate biomarker selection, Proteomics Clin Appl. 6 (2012) 297-303. https://doi.org/10.1002/prca.201100061.

[31] F. Mannello, Serum or plasma samples? The "Cinderella" role of blood collection procedures: preanalytical methodological issues influence the release and activity of circulating matrix metalloproteinases and their tissue inhibitors, hampering diagnostic trueness and leading to misinterpretation, Arterioscler. Thromb. Vasc. Biol. 28 (2008) 611-614. https://doi.org/10.1161/ATVBAHA.107.159608.

[32] R.B. Schnabel, S. Blankenberg, Commentary: Circulating cytokines and risk stratification of stroke incidence--will we do better in future?, Int J Epidemiol. 38 (2009) 261-262. https://doi.org/10.1093/ije/dyn263.

[33] Y. Yatomi, Y. Igarashi, L. Yang, N. Hisano, R. Qi, N. Asazuma, K. Satoh, Y. Ozaki, S. Kume, Sphingosine 1-phosphate, a bioactive sphingolipid abundantly stored in platelets, is a normal constituent of human plasma and serum, J. 
Biochem. 121 (1997) 969-973.

https://doi.org/10.1093/oxfordjournals.jbchem.a021681.

[34] J. Simon-Sanchez, S. Scholz, M. del M. Matarin, H.-C. Fung, D. Hernandez, J.R. Gibbs, A. Britton, J. Hardy, A. Singleton, Genomewide SNP assay reveals mutations underlying Parkinson disease, Hum. Mutat. 29 (2008) 315-322. https://doi.org/10.1002/humu.20626.

[35] H. Kobayashi, R. Krüger, K. Markopoulou, Z. Wszolek, B. Chase, H. Taka, R. Mineki, K. Murayama, O. Riess, Y. Mizuno, N. Hattori, Haploinsufficiency at the alpha-synuclein gene underlies phenotypic severity in familial Parkinson's disease, Brain. 126 (2003) 32-42. https://doi.org/10.1093/brain/awg010.

[36] K. Markopoulou, Z.K. Wszolek, R.F. Pfeiffer, B.A. Chase, Reduced expression of the G209A alpha-synuclein allele in familial Parkinsonism, Ann. Neurol. 46 (1999) 374-381.

[37] G.E. Voutsinas, E.F. Stavrou, G. Karousos, A. Dasoula, A. Papachatzopoulou, M. Syrrou, A.J.M.H. Verkerk, P. van der Spek, G.P. Patrinos, R. Stöger, A. Athanassiadou, Allelic imbalance of expression and epigenetic regulation within the alpha-synuclein wild-type and p.Ala53Thr alleles in Parkinson disease, Hum. Mutat. 31 (2010) 685-691. https://doi.org/10.1002/humu.21248.

[38] D.F. Lazaro, E.F. Rodrigues, R. Langohr, H. Shahpasandzadeh, T. Ribeiro, P. Guerreiro, E. Gerhardt, K. Krohnert, J. Klucken, M.D. Pereira, B. Popova, N. Kruse, B. Mollenhauer, S.O. Rizzoli, G.H. Braus, K.M. Danzer, T.F. Outeiro, Systematic comparison of the effects of alpha-synuclein mutations on its oligomerization and aggregation, PLoS Genetics. 10 (2014) e1004741. https://doi.org/10.1371/journal.pgen.1004741.

[39] J.E. Duda, B.I. Giasson, M.E. Mabon, D.C. Miller, L.I. Golbe, V.M.-Y. Lee, J.Q. Trojanowski, Concurrence of alpha-synuclein and tau brain pathology in the Contursi kindred, Acta Neuropathol. 104 (2002) 7-11. https://doi.org/10.1007/s00401-002-0563-3.

[40] A.M. Cuervo, L. Stefanis, R. Fredenburg, P.T. Lansbury, D. Sulzer, Impaired degradation of mutant alpha-synuclein by chaperone-mediated autophagy, Science. 305 (2004) 1292-5. https://doi.org/10.1126/science.1101738.

[41] T. Vogiatzi, M. Xilouri, K. Vekrellis, L. Stefanis, Wild type alpha-synuclein is degraded by chaperone-mediated autophagy and macroautophagy in neuronal cells, The Journal of Biological Chemistry. 283 (2008) 23542-56. https://doi.org/10.1074/jbc.M801992200.

\section{Figure Captions}

\section{Figure 1. Alpha-synuclein levels in GU-PD patients.}

Levels of (A) serum and (B) plasma $\alpha$-synuclein of GU-PD patients vs. healthy controls. (C) Correlation between $\alpha$-synuclein in serum and plasma. (D) ROC curve of $\alpha$-synuclein levels in serum

Data presented as mean $\pm 95 \%$ confidence interval; $* * p<0.01$ 
Figure 2. Alpha-synuclein levels in A53T-PD.

Levels of (A) serum and (B) plasma $\alpha$-synuclein of A53T mutation carriers vs. wildtype controls. Correlation between $\alpha$-synuclein in plasma and (C) UPDRS score or (D) disease duration.

Data presented as mean $\pm 95 \%$ confidence interval; ${ }^{*} \mathrm{p}<0.05,{ }^{*} p<0.01$

Figure 3. Absence of EDTA interference

(A) ELISA assay standard curve with and without EDTA by the measurement of recombinant $\alpha$-synuclein. (B) Measurement of a serum sample in absence and in presence of EDTA.

Data presented as mean $\pm 95 \%$ confidence interval 